\title{
ANÁLISIS DE LA PALEODIETA A PARTIR DE ISÓTOPOS ESTABLES DEL CARBONO Y DEL NITRÓGENO EN LA POBLACIÓN PREHISPÁNICA DE LA PUNA DE JUJUy (ARGENTINA)
}

\author{
María Laura Fuchs ${ }^{1}$, José Alberto Cocilovo y y Héctor Hugo Varela ${ }^{3}$
}

\section{* Introducción}

Resumen

El objetivo de este trabajo es establecer la dieta de los individuos puñenos en el período Tardío en su primera etapa de la vida. La muestra consistió en once piezas dentales (preferencialmente el tercer molar) pertenecientes a individuos adultos de las colecciones osteológicas de la Puna de Jujuy depositadas en el Museo Etnográfico y en el Museo de La Plata. Los resultados de $\delta^{13} \mathrm{C}$ indicaron que la mayoría de los individuos mantuvieron una dieta mixta y los valores del $\delta^{15} \mathrm{~N}$ se manifestaron elevados. Estos últimos pueden ser explicados por el uso de abono en los campos de cultivo o la aridez del clima. Los resultados fueron coherentes con los datos publicados para la misma región y para zonas aledañas, como San Pedro de Atacama.

Palabras claves: colecciones osteológicas - composición dietaria población puneña.

\begin{abstract}
The aim of this study is to analyze the diet of individuals in the first stage of life from the Puna of Jujuy during the Late Period. The sample consisted of eleven teeth (third molars preferentially) belonging to adults from osteological collections of the Puna de Jujuy housed in the Ethnographic Museum and the Museum of La Plata. $\delta^{{ }^{13}} \mathrm{C}$ results indicated that most of the individuals had a mixed diet with high $\delta^{15} \mathrm{~N}$ values. The latter can be explained by the arid climate or use of fertilizer in fields. The results were consistent with published data for the same region and neighboring areas, such as San Pedro de Atacama.
\end{abstract}

Key words: osteological collections - dietary composition puneña population.

Recibido: Octubre 2014. Aceptado: Octubre 2015
Se comienzan a utilizar los isótopos estables para explicar algunos problemas que surgen en la antropología y en la arqueología, y se aplican sobre restos óseos humanos. En sus inicios estaban preocupados por reconstruir la dieta de los individuos, pero luego las preguntas se fueron ampliando, como por ejemplo, determinar la duración de la lactancia materna, la residencia y los patrones de migración (Katzenberg 2008). A continuación daremos algunos ejemplos de las aplicaciones de los isótopos del carbono: establecer la dieta a través de los diferentes patrones fotosintéticos, plantas $\mathrm{C}_{3}$ y $\mathrm{C}_{4}$ (Vogel y van der Merwe 1977; Buikstra y Milner 1991; Ambrose y Norr 1993; Katzenberg et al. 1995; Ambrose y Buttler 1997); diferenciar entre una dieta marina y una terrestre (Ambrose y Butler 1997; Pate et al. 2001; Yesner et al. 2003; Coltrain et al. 2004). Algunos usos de los isótopos del nitrógeno son: distinguir los distintos niveles tróficos (Minagawa y Wada 1984; Schoeninger y DeNiro 1984; Heaton et al. 1986; Ambrose 1991), detección de recursos de agua dulce (Katzenberg y Weber 1999; Katzenberg 2008), como indicador de estrés hídrico (Sealy et al. 1987; Ambrose 1991; DeNiro 1987), como indicador de estrés proteínico (Katzenberg y Lovell 1999; White y Armelagos 1997), y también se pueden utilizar para hacer inferencias sobre la alimentación infantil y el destete (Fogel et al. 1989; Katzenberg 1999). Los isótopos de oxígeno y de estroncio se utilizan en estudios de residencia y migración (Schwarcz y Schoeninger 1991; Sealy et al. 1991; White et al. 1998; Knudson et al. 2004).

1 Departamento de Ciencias Naturales. Facultad de Ciencias Exactas, Físico-Química y Naturales, Universidad Nacional de Río Cuarto. Ruta Nacional 36, Km. 601 (Código Postal X5804BYA), Río Cuarto, ARGENTINA. Email: mfuchs@exa.unrc.edu.ar

2 Departamento de Ciencias Naturales. Facultad de Ciencias Exactas, Físico-Química y Naturales, Universidad Nacional de Río Cuarto. Ruta Nacional 36, Km. 601 (Código Postal X5804BYA), Río Cuarto, ARGENTINA. Email: jcocilovo@exa.unrc.edu.ar

3 Departamento de Ciencias Naturales. Facultad de Ciencias Exactas, Físico-Química y Naturales, Universidad Nacional de Río Cuarto, Ruta Nacional 36, Km. 601 (Código Postal X5804BYA), Río Cuarto, ARGENTINA. Email: hvarela@exa.unrc.edu.ar 
Para la región que nos interesa estudiar, Puna de Jujuy, Argentina, los primeros trabajos isotópicos comienzan con Fernández y Panarello (1999-2001a) que realizaron análisis sobre animales silvestres de ambientes altiplánicos de la Argentina. Se obtuvieron muestras de pelo de distintos animales para evaluar la composición isotópica del carbono y del nitrógeno. En un trabajo posterior, Fernández y Panarello (1999-2001b) estudiaron los isótopos estables del carbono de muestras de colágeno óseo de herbívoros y carnívoros, modernos y prehistóricos, de la puna jujeña. La muestra siguió la cadena trófica (vegetal, herbívoro y carnívoro) y consistió en 45 especies de vegetales representativas de la flora altoandina y puneña, 37 de colágeno de herbívoros, ocho de carnívoros modernos y seis muestras de hueso arqueológico de camélidos provenientes de un montículo de Pozuelos. Los resultados establecieron una clara tendencia en el empobrecimiento de los valores de $\delta^{13} \mathrm{C}$ a medida que se aumenta en altitud, manifestándose por la distribución diferencial de las especies $\mathrm{C}_{3}$ y $\mathrm{C}_{4}$ de acuerdo a la altitud.

La dieta desde el Holoceno Temprano hasta los momentos posthispánicos del noroeste argentino fue estudiada por Olivera y Yacobaccio (1999), a través del análisis de isótopos estables, utilizando una muestra de 12 individuos, distribuidos cronológicamente en tres períodos. Establecieron tres tipos de dietas diferentes, según la relación del $\delta^{{ }^{13}} \mathrm{C}$ del colágeno y del carbonato y dos grandes conjuntos en la cadena trófica, a través de la relación isotópica de los valores del carbono y del nitrógeno. De esta última relación obtuvieron dos grupos; el primero corresponde a las plantas $\mathrm{C}_{4} \mathrm{e}$ individuos con dieta basada en maíz, mientras que el segundo se distribuye dentro de los herbívoros, incluyendo a los individuos con dieta rica en proteínas animales y plantas $C_{3}$. Para el caso de la Puna de Jujuy, su comportamiento se acercaría al del segundo grupo incluyendo una dieta rica en proteínas animales y en plantas $C_{3}$. En la Puna, las plantas $C_{3}$ incluyen a la mayoría de las plantas silvestres, a la quínoa, porotos, papa, oca, ulluco y mashua (Olivera y Yacobaccio 1999; Albeck y Ruiz 2003). Esta tendencia habría sido observada por Fernández y Panarello (1999-2001b) para los camélidos arqueológicos del sitio de Pozuelos, los valores de $\delta^{13} \mathrm{C}$ promedian un valor de $-16.5 \%$ configurando una dieta con el $65 \%$ de plantas $\mathrm{C}_{3}$ y el $35 \%$ de plantas $\mathrm{C}_{4}$, con una cronología comprendida entre $745 \pm$ 45 y $455 \pm 50$ años AP.
Posteriormente, se utilizaron los valores aportados por los isótopos estables de carbono y nitrógeno sobre el colágeno óseo de camélidos actuales y arqueológicos para determinar las estrategias de pastoreo y movilidad de los pastores y compararlos con los datos etnoarqueológicos (Yacobaccio et al. 2009; Yacobaccio et al. 2010). Los resultados indicaron una clara tendencia entre los valores de $\delta^{13} \mathrm{C}$ y la altitud, pues a medida que aumenta la altitud, los valores de $\delta^{13} \mathrm{C}$ se hacen más negativos. Esta tendencia les permite afirmar que la dieta de los camélidos puneños, tanto domésticos como silvestres, manifiestan mayor importancia de las especies $\mathrm{C}_{3}$ en comunidades de altura, mientras que las especies $\mathrm{C}_{4}$ cobrarían relevancia en ambientes de menor altitud.

En el trabajo de Pérez y Killian Galván (2011) y Killian Galván et al. (2012) se presentan los resultados del análisis tecnoestilístico de recipientes cerámicos y la inferencia paleodietaria a través de los isótopos estables de carbono y nitrógeno sobre restos óseos humanos del área arqueológica del río Doncellas. Los resultados arrojaron valores de $\delta^{13} \mathrm{C}$ co que se ajustan a los consumidores de una dieta mixta, entre $-17 \%$ y $-14 \%$, registrándose un consumo bajo o indirecto de $\mathrm{C}_{4}$. Con respecto a los valores de $\delta^{15} \mathrm{~N}$ éstos indican que las dietas de los individuos tuvieron un alto aporte cárnico.

Como se resumió anteriormente, la información isotópica sobre restos óseos humanos en la región de la Puna de Jujuy es muy acotada. Por lo tanto, en este trabajo pretendemos realizar un análisis exploratorio de los isótopos de carbono y del nitrógeno de los grupos humanos puneños en el período Tardío y compararlos con los resultados publicados para la misma región y zonas aledañas. Teniendo en cuenta las referencias antes mencionadas, en la presente experiencia se espera que los individuos puneños manifiesten una dieta rica en proteínas animales, de origen terrestre, y plantas $C_{3}$.

\section{La Puna de Jujuy en el período Tardío (1000-1450 DC)}

Al norte y al oeste de la provincia de Jujuy (Argentina) está ubicada la Puna de Jujuy, que constituye una alta meseta con altitudes variables entre 3500 y 4500 metros y sus cordones montañosos se desarrollan de norte a sur con picos de más de 6000 metros. Además presenta 
amplias depresiones, cuencas sedimentarias de drenaje endorreico rodeadas por sierras que contienen salares o lagunas importantes como la de Los Pozuelos, Vilama y Miraflores-Guayatayoc-Salinas Grandes. Su geografía incluye también cuencas de drenaje atlántico como las del río Grande de San Juan, Yavi y Sansana, que desembocan en el río Pilcomayo (Albeck y Ruiz 2003).

Los grupos humanos que vivieron en la Puna en el período Tardío se dedicaban a la actividad ganadera, tanto para su consumo local como bien de intercambio, mientras que las prácticas agrícolas se restringían a aquellas áreas con disponibilidad de agua, que favorecían el riego. También se dedicaron a la extracción de sal, metales y rocas, que les sirvieron para el intercambio con regiones aledañas (Albeck y Ruiz 2003).

Entre los sitios arqueológicos más característicos encontramos al Pucará de Rinconada, ubicado al sudoeste de la cuenca de Pozuelos sobre un macizo elevado en donde se localizan las ruinas de un antiguo poblado, un conglomerado con defensas (Lehmann Nistche 1902; Boman 1908; Madrazo y Ottonello de García Reinoso 1966). Posee recintos de planta rectangular que se agrupan formando un patio central, y en las paredes de algunos recintos aparecen integrados trozos de menhires. Conserva un sistema de canaletas o entubamientos subterráneos que probablemente sirvieron para el drenaje de las aguas meteóricas. Además, se hallaron sepulcros construidos con paredes de barro, presencia de inhumaciones de varios individuos superpuestos y varios entierros directos (Alfaro y Suetta 1970; Albeck y Ruiz 2003).

Agua Caliente de Rachaite (Ottonello de García Reinoso 1973), también conocido como Doncellas (Alfaro y Suetta 1976), se trata de un asentamiento que se extiende sobre ambos márgenes de un curso temporario de agua, afluente del río Rachaite. Fue definido como un poblado agrícola estable de tipo conglomerado, con predominio de recintos asociados desiguales y un tipo simple de recinto perimetral compuesto (Madrazo y Otonello de García Reinoso 1966). Los enterratorios ocupan la parte inferior de los farellones y la parte superior de las laderas, predominando dos tipos de inhumación: en huecos naturales que son cerrados por muros de piedra y barro, y las tumbas de tipo chullpas o casas tumba. Sobre el área contigua al poblado, se distribuyen varias terrazas y cuadros de cultivo (Ottonello de García Reinoso 1973). Posteriormente, Alfaro $(1983,1988)$ realiza nuevas excavaciones dando cuenta de la arquitectura civil, constituida por viviendas de recintos de forma cuadrangular o rectangular, círculos hundidos o habitaciones semisubterráneas, andenes de cultivo y acequias. A su vez, describe una arquitectura religiosa centrada en una estructura escalonada, con una posición estratégica y la presencia de menhires. Para la arquitectura funeraria define cuatro tipos de enterratorios: casas tumba, grutas tapiadas, sepulcros de planta semicircular y sepulcros de planta circular o redonda, con distintas formas de inhumación.

El sitio Queta (15 km hacia el noroeste de Doncellas) ha sido citado por arqueólogos que efectuaron investigaciones o visitaron el sitio, pero sólo se sabe lo dicho por Boman (1908) y Madrazo y Ottonello (1966), que lo definen como un poblado de tipo conglomerado sin defensa.

En marzo de 1944, Casanova informa al Museo de Ciencias Naturales sobre las excavaciones llevadas a cabo en Queta y pone al descubierto un gran número de casas, entierros de esqueletos sin ajuar y la presencia de grandes ollas vacías y quebradas. Posteriormente, Alfaro de Lanzone (1988) recorre este sitio, del cual apenas quedaban pedazos, y solo halla una cista parcialmente abierta, tres urnas utilitarias con restos de párvulos. Del material recolectado en superficie, encuentra cuentas de piedra toscamente talladas conocidas como "cuentas de Queta".

El sitio Agua Caliente se ubica a $15 \mathrm{~km}$ hacia el oeste de Doncellas, sobre la misma línea que este sitio pero en la margen opuesta del río. Se trata de un conglomerado o semiconglomerado sin defensas, recintos rectangulares y cuadrangulares formando unidades simples y compuestas (Alfaro 1983). El área de Casabindo está comprendida por una serie de quebradas con recursos de agua permanente y presenta restos arqueológicos vinculados a las prácticas agrícolas prehispánicas. Se postulan tres grandes áreas de cultivo para la zona de Casabindo: Sayate, Potrero-Tarante y Río Negro, en los cuales se pueden observar antiguos terrenos de cultivo (Albeck 2011). Hay varios asentamientos arqueológicos entre ellos: Pueblo Viejo de Potrero, Ojo de Agua, Calaverioj (Albeck y Ruiz 2003). 
Al suroeste del poblado actual de Casabindo se encuentra el sitio Pueblo Viejo de Tucute (Albeck 1999; Albeck y Ruiz 2003) o Sorcuyo (Casanova 1938), ubicado sobre el tramo inferior del arroyo de Tucute en la Quebrada de Tucute. Se trata de un extenso poblado de tipo semiconglomerado que ocupa dos lomadas ubicadas a ambos lados del arroyo. Las viviendas son de planta circular levantadas con piedras canteadas en forma prismática de roca volcánica. Dentro de los materiales cerámicos encontrados se destacan dos grupos formales, vasijas no restringidas (pucos, platos y vasitos chatos) y vasijas restringidas con cuello, a estos se suman vasijas zoomorfas con modelados de llama. La cerámica es de tipo ordinaria y sólo algunas aparecen pintadas con una pintura roja muy liviana. El material lítico comprende piedras de moler, manos, pulidores y puntas de flecha triangulares de base escotada. Posee un rango temporal que abarca desde fines del 900 a principios del $1500 \mathrm{AD}$ (Albeck y Zaburlín 2008).

\section{* Materiales y métodos}

La muestra consistió en once piezas dentales pertenecientes a once individuos adultos de la población tardía (Fuchs y Varela 2013) de la Puna de Jujuy. Las respectivas piezas dentales pertenecen a restos óseos humanos depositados en el Museo etnográfico Juan B. Ambrosetti (Facultad de Filosofía y Letras, Universidad de Buenos Aires) y en el Museo de La Plata (Facultad de Ciencias Naturales y Museo, Universidad Nacional de La Plata). La muestra del Museo etnográfico está compuesta por nueve piezas dentales provenientes de las localidades de Doncellas, Agua Caliente, Río Negro, Sorcuyo, Casabindo y Queta (Figura 1), según el Catálogo del Depósito de Antropología Biológica, mientras que la muestra del Museo de La Plata fue de dos piezas dentales provenientes del Cementerio VIII de Casabindo y del Cementerio II del Pucará de Rinconada (Figura 1).

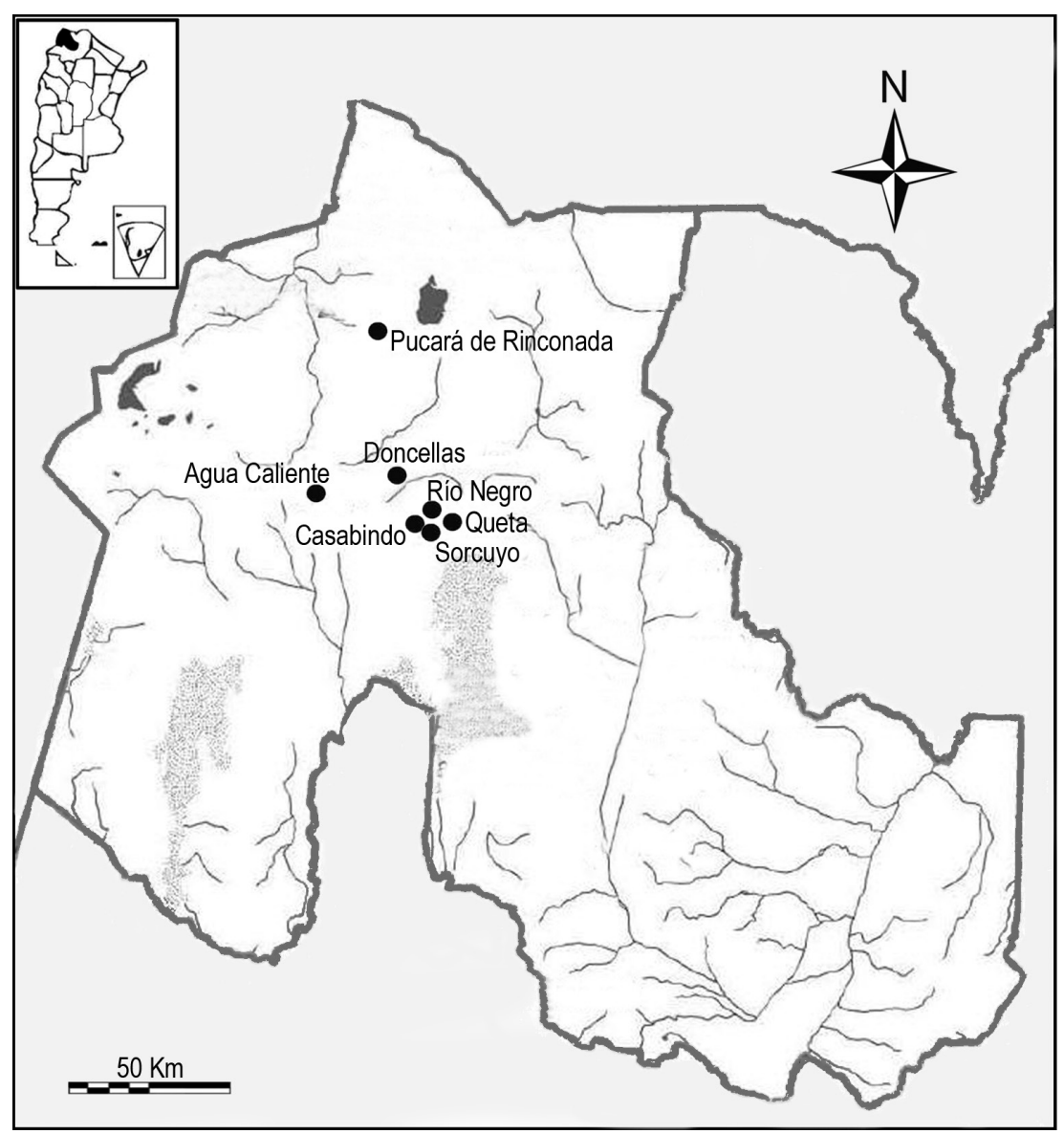

Figura 1. Localización de los sitios. 
La estrategia de selección de la muestra fue la extracción preferencial de los terceros molares, dado que la primera evidencia de calcificación comienza a los siete u ocho años, la corona está completa entre los 12 y 16 años y la raíz se completa entre los 18 y 25 años. En su defecto se extrajeron los segundos molares, cuya corona está completa entre los siete y ocho años y la raíz entre los 14 y 16 años (Nelson y Ash 2010). Se seleccionaron sólo individuos adultos, para no tener sesgos con respecto a la edad y de ambos sexos. Estos dientes representan la dieta que tuvieron estos individuos en su infancia y adolescencia antes de pasar a ser adultos en su residencia local. Los dientes tienen etapas de formación según la edad de los individuos y brindan información sobre las distintas dietas antes y después del destete. Los valores de $\delta_{15} \mathrm{~N}$, medidos sobre el colágeno de la dentina, corresponden en su totalidad a las proteínas de la dieta (van der Merwe et al. 2003).

Se determinaron los valores de $\delta^{13} \mathrm{C}, \delta^{15} \mathrm{~N}$ y radiocarbónicos a partir del colágeno de los dientes (dentina + esmalte) y fueron realizados por el laboratorio de física de la Universidad de Arizona, NSF Arizona AMS Facility, con un espectrómetro de masas con el sistema NEC 3MV Pelletron. Las muestras de dientes fueron limpiadas empleando vibración por ultrasonido y agua, se secaron y luego fueron trituradas en un mortero de porcelana $y$ con una mano de molido de 0,5 a $1 \mathrm{~mm}$. Estos fueron cargados en celdas de flujo continuo a través del cual se bombeó una secuencia programada de lavados que contienen agua desionizada, o,1 $\mathrm{M} \mathrm{HCl,} \mathrm{o,5} \mathrm{M} \mathrm{NaOH} \mathrm{y} \mathrm{o,ol}$ $\mathrm{M} \mathrm{HCl}$, durante 16 horas. Los fragmentos desmineralizados fueron gelatinizados en tubos de ensayo a $70{ }^{\circ} \mathrm{C}$ durante una noche y luego filtrados a través de una fibra de vidrio de o, 45 ctm. El colágeno se recuperó de los filtrados por liofilización.

Las tasas de isótopos estables del carbono $\left({ }_{13} \mathrm{C} / 12 \mathrm{C}\right)$ se expresan como $\delta_{13} \mathrm{C}$ y son utilizadas como un índice de la importancia relativa de las plantas $\mathrm{C}_{3}, \mathrm{C}_{4}$ y CAM en la dieta. Los resultados se expresan en partes por mil (\%o) relativas al estándar internacional PDB (Pee Dee Belemnite Carbonate) con un error menor al o,1\%o. Las plantas $\mathrm{C}_{3}$ utilizan la vía fotosintética Calvin Benson y poseen valores bajos de $\delta_{13} \mathrm{C}$ alrededor del $-20 \%$ al $-34 \%$, con una media de $-26 \%$. Las plantas $\mathrm{C}_{4}$ emplean la vía fotosintética Hatch-Slack y tiene valores de $\mathrm{\delta}_{13} \mathrm{C}$ alrededor del -9\% al -16\%, con un media de $-12 \%$ (Ambrose 1993; Panarello et al. 2006-2009).

Las plantas llamadas CAM utilizan las dos vías fotosintéticas mencionadas anteriormente según las condiciones ambientales imperantes. Si el carbono es fijado durante el día, se asemejan a las plantas $\mathrm{C}_{3}$, con valores de $\delta^{13} \mathrm{C}$ alrededor de -24 a $-30 \%$. Cuando el carbono se fija durante la noche, los valores oscilan entre -10 y -14\%o, asemejándose a las plantas C4 (Ambrose 1993; Pate 1994). Los valores de $\delta^{13} \mathrm{C}$ del colágeno reflejan el componente proteico de la dieta y su tasa de enriquecimiento es de 3\% a 5\% en los tejidos (Ambrose 1993; Tikot 2006).

Por otra parte, los isótopos del nitrógeno se presentan de dos formas en la naturaleza $(15 \mathrm{~N} / 14 \mathrm{~N})$, se expresan como $\delta^{15} \mathrm{~N}$ en partes por mil (\%o) según el estándar internacional (Air) con un error menor al o,2\%o. La obtención del nitrógeno en las plantas terrestres no es directa, sino que puede adquirirse en relación simbiótica con otros organismos o a partir de las bacterias que viven en el suelo y los valores de $\delta^{15} \mathrm{~N}$ están entre -2\%o y 6\%o (Pate 1994). A medida que ascendemos en la cadena trófica, la tasa de enriquecimiento es del 3-4\%o (Ambrose 1993; Pate 1994). Los herbívoros pueden tener valores $\delta^{15} \mathrm{~N}$ entre el 1 al 12,7\%o (promedio: 5,28\% $\pm 2,6 \%$ ), mientras que los carnívoros tienen valores entre $5,3 \%$ a $18,8 \%$ (promedio: 10,2\% \pm 2,9\%) (Panarello et al. 2006-2009). Dado que las proporciones de los isótopos del nitrógeno aumentan entre un nivel trófico y otro, nos proporciona una medida del consumo de proteína animal y nos permite distinguir entre recursos de origen marino o terrestre (Ambrose et al. 2003; Panarello et al. 2006-2009).

\section{$*$ Resultados}

En la tabla 1 se detalla la procedencia, el número de catálogo, el código del laboratorio, el tipo de material extraído, los resultados de $\delta^{13} \mathrm{Cco}$ y $\delta^{15} \mathrm{Nco}$ y las fechas obtenidas.

En la tabla 2 se presenta la estadística descriptiva de la muestra. En ella se consigna el número de individuos, la media, el desvío estándar, el valor mínimo y máximo, la mediana, el primer y tercer cuartil del $\delta^{13} \mathrm{Cco}$ y del $\delta^{15} \mathrm{Nco}$. El $\delta^{13}$ Cco presenta una media de $-16,56 \%$, 


\begin{tabular}{|c|c|c|c|c|c|c|}
\hline Procedencia & No catálogo & Cód. Lab. & Material & $\delta^{13} C_{c o}$ & $\delta^{15} \mathrm{~N}_{c o}$ & ${ }^{14} \mathrm{C} \mathrm{BP}$ \\
\hline Casabindo (ME) & 14136 & AA89343 & $3 \mathrm{MSD}$ & -17.9 & - & $509 \pm 42$ \\
\hline Doncellas (ME) & 13955 & AA89344 & $3 \mathrm{MSI}$ & -18.5 & - & $568 \pm 44$ \\
\hline Doncellas (ME) & 13958 & AA89345 & $3 \mathrm{MSD}$ & -17.2 & - & $585 \pm 42$ \\
\hline Agua Caliente (ME) & 15438 & $\mathrm{AA} 94988$ & $2 \mathrm{MSI}$ & -18.6 & 12.7 & $559 \pm 41$ \\
\hline Doncellas (ME) & 13937 & AA94991 & $3 \mathrm{MSI}$ & $-17 \cdot 3$ & 13.1 & $602 \pm 33$ \\
\hline Río Negro (ME) & 14127 & AA94989 & $3 \mathrm{MSI}$ & -15.5 & 12.2 & $643 \pm 33$ \\
\hline Sorcuyo (ME) & 14178 & AA94990 & 2MSI & -16.2 & 13 & $633 \pm 38$ \\
\hline Casabindo (ME) & 14149 & AA94992 & 2MSI & $-15 \cdot 4$ & 13.4 & $615 \pm 33$ \\
\hline Queta (ME) & 14194 & AA94993 & $3 \mathrm{MSI}$ & -15.9 & 12.1 & $665 \pm 33$ \\
\hline Casabindo (MLP) & 448 & $\mathrm{AA} 98245$ & $3 \mathrm{MSI}$ & -13.7 & 12.9 & $501 \pm 42$ \\
\hline Pucara de Rinconada (MLP) & 457 & $\mathrm{AA} 98246$ & 3MII & -16 & 13.5 & $964 \pm 45$ \\
\hline
\end{tabular}

Tabla 1. Detalles de la muestra y resultados de los isótopos.

\begin{tabular}{|l|c|c|}
\hline Variable & $\delta^{13} \mathrm{C}_{\mathrm{co}}$ & $\delta^{15} \mathrm{~N}_{\mathrm{co}}$ \\
\hline $\mathrm{N}$ & 11 & 8 \\
\hline Media & -16.56 & 12.86 \\
\hline D.E. & 1.49 & 0.51 \\
\hline Valor Mínimo & -18.6 & 12.1 \\
\hline Valor Máximo & -13.7 & 13.5 \\
\hline Mediana & -16.2 & 12.95 \\
\hline Q1 & -17.9 & 12.2 \\
\hline $\mathrm{Q}_{3}$ & -15.5 & 13.1 \\
\hline
\end{tabular}

Tabla 2. Estadística descriptiva de la muestra.

con un valor mínimo de $-18,6 \%$ y un valor máximo de $-13,7 \%$. Mientras que el $\delta^{15} \mathrm{Nco}$ presenta una media de $12,86 \%$, con un valor mínimo de $12,1 \%$ y un máximo de $13,5 \%$.

En la figura 2 se visualiza la relación entre el $\delta^{13} \mathrm{Cco}$ y el $\delta^{15} \mathrm{Nco}$ para cada procedencia. Las líneas de corte representan los valores medios de los isótopos del carbono y del nitrógeno. Para la muestra de $\delta^{15} \mathrm{Nco}$, los valores más elevados corresponden a Casabindo, Doncellas y Rinconada, un valor intermedio para Agua Caliente y los valores más bajos los presentan Río Negro y Queta. Para el caso de la muestra del $\delta^{13} \mathrm{Cco}$, los valores más elevados corresponden a Casabindo, Río Negro, Sorcuyo, Queta y Rinconada, mientras que los más bajos son Doncellas y Agua Caliente.

Los sitios Sorcuyo (633 $\pm 38 \mathrm{BP})$, Queta $(665 \pm 33 \mathrm{BP})$, Río Negro $(643 \pm 33 \mathrm{BP})$ y un individuo de Casabindo $(615 \pm 33 \mathrm{BP})$, presentan valores de $\delta^{13} \mathrm{Cco}$ de alrededor del -16,1\%. Los sitios Agua Caliente (559 $\pm 41 \mathrm{BP})$, Doncellas (del 568 al 602 BP) y Casabindo (509 \pm 42 BP) presentan valores de $\delta^{13} \mathrm{Cco}$ menos enriquecidos (ver Tabla 1). Los valores de $\delta_{13}$ Cco en relación al tiempo parecen mostrar una tendencia hacia un menor enriquecimiento en los momentos más tardíos, mientras que los valores de $\delta^{15} \mathrm{Nco}$ en relación al tiempo parecen no manifestar ninguna tendencia y sus valores se encuentran arriba del $12 \%$.

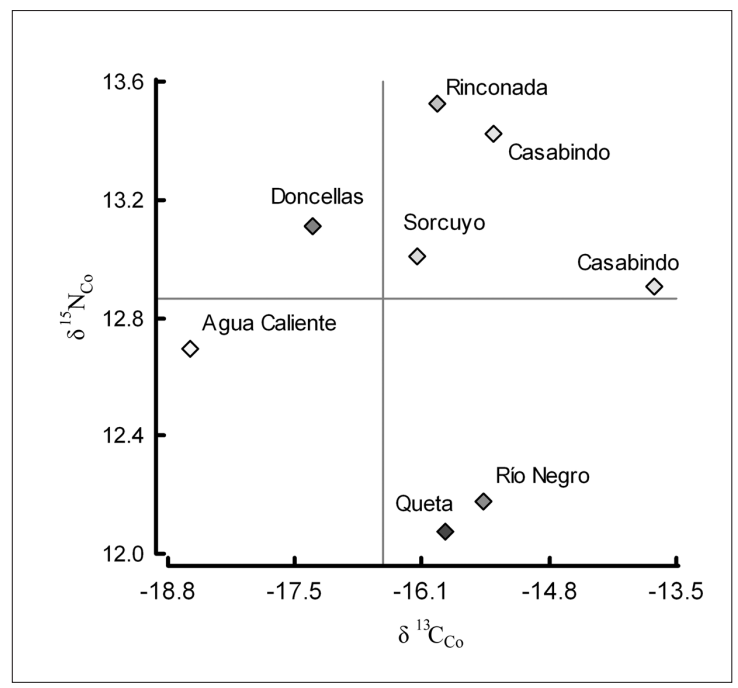

Figura 2. Relación entre el $\delta^{13} \mathrm{Cco}$ y el $\delta^{15} \mathrm{Nco}$. 


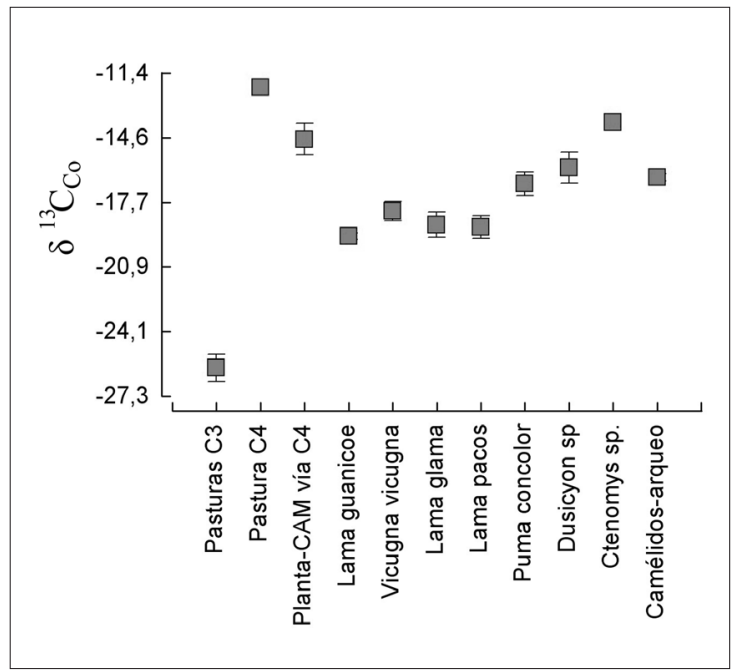

Figura 3. Valores de $\delta_{13} \mathrm{Cco}$ de especies vegetales y animales de la región (sacado de Fernández y Panarello 1999-2001b).

El Pucará de Rinconada arrojó la fecha más temprana: $964 \pm 45$ BP. Posee un valor de $13,5 \%$ de $\delta^{15} \mathrm{Nco}$ y un valor de -16\% de $\delta^{13} \mathrm{Cco}$. Para el Cementerio VIII de Casabindo, con $501 \pm 42 \mathrm{BP}$, posee un valor de $\delta^{15} \mathrm{Nco}$ de $12,9 \%$ y de $\delta^{13} \mathrm{C}$ co de $-13,7 \%$.

\section{* Discusión}

Como se mencionara anteriormente, los resultados obtenidos en este trabajo representan la dieta que tuvieron los individuos puneños en su infancia y adolescencia antes de pasar a ser adultos en su residencia local. Esta información es de suma importancia, ya que los individuos que vivieron en la Puna de Jujuy se caracterizaron por sus prácticas de caravaneo, por sus productos de intercambio y su alta movilidad entre la región y zonas aledañas. En una experiencia etnográfica realizada por Axel Nielsen con un grupo de pastores del sur de Lipez, en donde participa como uno de los ayudantes en un viaje de larga distancia con caravanas de llamas a los valles orientales, observa que esta actividad es fundamentalmente masculina. Además, la caravana está constituida por dos o tres hombres que empiezan a viajar a partir de los 12 años como ayudantes. A esa edad, tanto hombres como mujeres asumen la responsabilidad de ocuparse en las actividades productivas de la unidad doméstica (Nielsen 1997-1998).

Los resultados obtenidos de los valores de $\delta^{13} \mathrm{Cco}$ de la mayoría de los individuos de la Puna de Jujuy indican que se habría mantenido una dieta mixta (-17\%o y -14\%o, Coltrain y Leavitt 2002), el consumo alimenticio correspondería a plantas $\mathrm{C}_{3}$ y $\mathrm{C}_{4}$. Este patrón concuerda con los valores aportados por la ecología isotópica de la región que se resume en la figura 3. Otros individuos presentan valores de $\delta^{13} \mathrm{C}$ más empobrecidos, como es el caso de aquellos procedentes de Casabindo ( $\mathrm{N}^{\circ}$ cat. 14136), Doncellas (13955) y Agua Caliente (15934), que manifestarían la influencia de recursos $C_{3}$. Además hay un individuo procedente de Casabindo ( $\mathrm{N}^{\circ}$ cat. 448 ) que presenta un valor enriquecido de $\delta^{13} \mathrm{C}$ con respecto al resto de la muestra, que mostraría la influencia de los recursos $\mathrm{C}_{4}$.

Nuestros resultados son consistentes con los obtenidos en los trabajos de Pérez y Killian Galván (2011) y Killian Galván et al. (2012), en donde se analiza una muestra de la localidad arqueológica del río Doncellas. Estos trabajos utilizaron muestras de hueso (preferentemente costillas) para obtener los valores isotópicos y representan la dieta que tuvieron esos individuos en la última década su vida. En las figuras 4 y 5 podemos observar cómo se manifiestan los valores de $\delta^{13} \mathrm{Cy} \delta^{15} \mathrm{~N}$ en el tiempo, tanto para los individuos de la localidad de Río Doncellas (sólo los individuos que poseen fechas) como los aquí presentados.

Al observar los valores de $\delta^{13} \mathrm{C}$ en el tiempo (Figura 4), podemos decir que los individuos procedentes del Pucará de Rinconada (964 \pm 45 AP), Río Negro (643 \pm 33 AP), Queta $(665 \pm 33 \mathrm{AP})$ y Sorcuyo $(633 \pm 38 \mathrm{AP})$ habrían

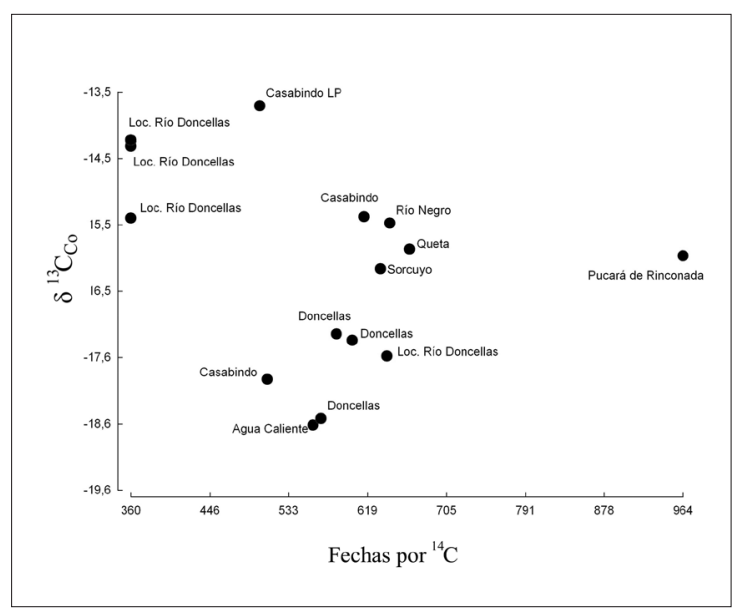

Figura 4. Tendencias temporales de los valores de $\delta^{13} \mathrm{C}$ en los distintos sitios y de la loc. Río Doncellas (Pérez y Killian Galván 2011). 
mantenido una dieta mixta. En el sitio arqueológico Pueblo Viejo de Tucute o Sorcuyo se recuperaron restos de papa y maíz carbonizado y restos óseos correspondientes a camélidos (Albeck 1999; Albeck y Ruiz 2003). Asimismo, en el sitio arqueológico Pozuelos se encontraron restos de camélidos arqueológicos y los valores de $\delta^{13} \mathrm{C}$ obtenidos de sus huesos configuraban una dieta mixta (Fernández y Panarello 1999-2001b).

Los tres individuos procedentes de Casabindo manifiestan tres tipos de dietas: el primer individuo (615 $\pm 33 \mathrm{AP})$ tuvo una dieta mixta, el segundo ( $501 \pm 42 \mathrm{AP})$ manifiesta la influencia de los recursos $\mathrm{C}_{4}$, y por último, el tercero (509 $\pm 42 \mathrm{AP}$ ) revela la influencia de los recursos $\mathrm{C}_{3}$ en su dieta. Los individuos procedentes de Doncellas y Agua Caliente, que se encuentran alrededor del 1398 al 1451 AD, y un individuo de la localidad de Río Doncellas (Don 138, 640 $\pm 50 \mathrm{AP}$ ) manifiestan valores de $\delta^{13} \mathrm{C}$ empobrecidos que nos indicarían la influencia de los recursos $C_{3}$. Hay tres individuos que se ubican alrededor del $360 \pm 50 \mathrm{AP}$, como es el caso de aquellos que pertenecen a la localidad de Río Doncellas (Don 88, Don 215 y Don 224), que manifiestan una dieta mixta y poseen un contexto asociado a la ocupación incaica (Pérez y Killian Galván 2011). Resumiendo, podemos decir que desde los momentos tempranos hasta los más tardíos de la muestra, la mayoría de los individuos puneños habrían mantenido una dieta mixta y algunos individuos manifestarían otro tipo de dieta, pero debemos considerarlos como una excepción.

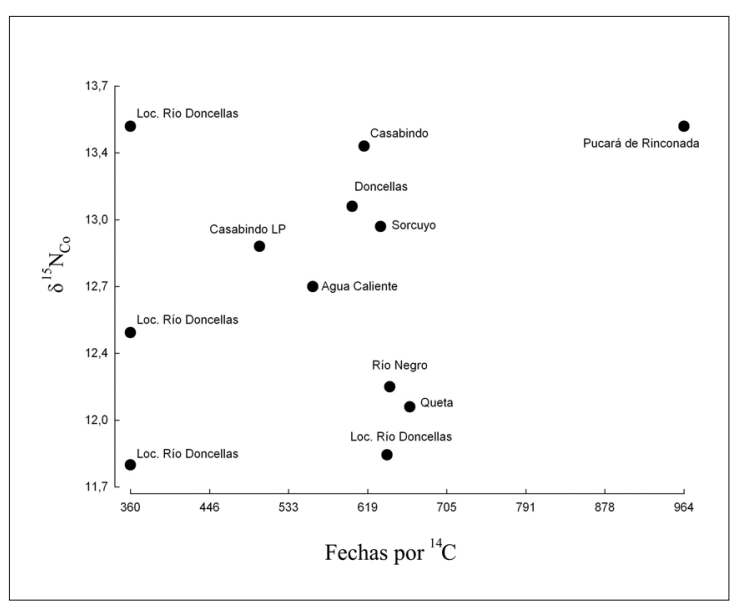

Figura 5. Tendencias temporales de los valores de $\delta^{15} \mathrm{Nco}$ en los distintos sitios y de la loc. Río Doncellas (Pérez y Killian Galván 2011).
En cuanto a los valores del $\delta^{15} \mathrm{~N}$ en el tiempo (Figura 5), no se observa ninguna tendencia y los valores son elevados en los distintos sitios. Una de las propiedades del isótopo del nitrógeno nos permite distinguir entre el consumo de recursos marinos y terrestres. Sin embargo, para nuestro caso particular, el registro zooarqueológico de los sitios estudiados no registra restos de fauna marina, todo lo contrario, hay registro de restos de camélidos (Albeck 1998). En el área puneña es muy característica la utilización de las llamas para el transporte y el movimiento de mercancías de un lugar a otro. Por lo tanto, la llama está sujeta al manejo humano, y sus territorios de alimentación pueden variar según la abundancia de pasturas $\mathrm{C}_{3}$ y $\mathrm{C}_{4}$ en las diferentes zonas y según la altitud del pastoreo. A su vez, la llama es un mamífero tolerante a la sequía y presenta valores del $\delta^{15} \mathrm{~N}$ más elevados que otros animales, estos valores se asemejan a los esperados para los ambientes desérticos (Yacobaccio et al. 2010).

Los valores de nitrógeno enriquecidos de los individuos puneños puede deberse al consumo de plantas cultivadas que fueron abonadas con fertilizantes orgánicos o al consumo de herbívoros con distintos patrones alimenticios. Además pueden intervenir otros factores como las inclemencias del clima, la aridez, la altitud y las precipitaciones (Schwarcz et al. 1999). Varios autores han demostrado que $\delta^{15} \mathrm{~N}$ es muy sensible al clima y a los ambientes áridos, manifestándose en valores elevados (Heaton et al. 1986; Ambrose 1991). Se han realizado experiencias en animales en donde se compara la misma especie pero de ambientes áridos y de ambientes más húmedos, y los resultados muestran que el $\delta^{15} \mathrm{~N}$ varía de una región a otra (Heaton et al. 1986; Sealy et al. 1987). Los estudios relacionados con los mecanismos fisiológicos involucrados en la variación de los valores de $\delta^{15} \mathrm{~N}$ en mamíferos que viven en regiones áridas, proponen un modelo explicativo de pérdida de nitrógeno a través de la urea que se excreta por la orina (DeNiro 1987; Ambrose 1991). Estos autores plantean que, en condiciones de estrés hídrico, el isótopo más ligero ${ }_{14} \mathrm{~N}$ se pierde a través de la orina y el ${ }_{15} \mathrm{~N}$ es retenido por el cuerpo, y este se encuentra disponible para ser sintetizado por los tejidos (DeNiro 1987; Ambrose 1991).

Se ha comprobado que el uso de fertilizantes orgánicos, como el guano de camélidos y el de aves marinas, tiene un impacto sobre los valores del isótopo del nitrógeno 
en las plantas. Hace unos años, se llevó a cabo un experimento con cultivos de plantas de maíz que fueron fertilizadas con cuatro tratamientos (control, fertilizante químico, estiércol de camélidos y guano de aves marinas). Los resultados indicaron que las plantas fertilizadas con guano de aves marinas enriquecieron notablemente los valores de nitrógeno; del mismo modo, las plantas fertilizadas con estiércol de camélidos arrojaron valores más altos que los observados para el control (Szpak et al. 2012). En otro trabajo se estudiaron los isótopos estables $(\mathrm{N}, \mathrm{C})$ del cálculo dental de sitios arqueológicos de la costa y del valle del norte de Chile desde el período Arcaico hasta el Intermedio Tardío. Los resultados arrojaron que los valores $\delta^{15} \mathrm{~N}$ de las poblaciones costeras eran más elevados que los de los habitantes de los valles, y sugieren como posible explicación el uso de guano de aves marinas como fertilizante (Poulson et al. 2013). En la región de Antofagasta de la Sierra, Catamarca (Argentina), se estudió la relación entre el uso de fertilizantes y riego, y sus consecuencias en la ecología isotópica de tres especies (Zea mays, Chenopodium quinoa y Solanum tuberosum) de plantas cultivadas (Killian Galván y Salminci 2014). Los resultados arrojaron un amplio rango de valores de $\delta^{15} \mathrm{~N}$ para el maíz, algunos especímenes se mostraron enriquecidos y fueron consistentes con los valores obtenidos en muestras de restos óseos humanos para la Puna de Jujuy.

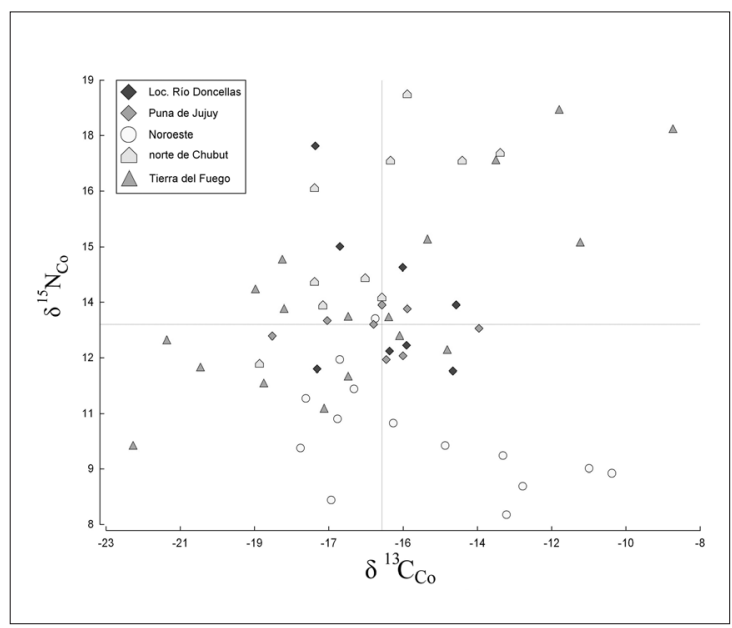

Figura 6. Representación gráfica de los valores de $\delta^{13} \mathrm{C}$ y $\delta^{15} \mathrm{~N}$ de distintos sitios arqueológicos de la Argentina: Puna de Jujuy (muestra actual y de la Loc. Arqueo. Río Doncellas, Pérez y Killian Galván 2011); noroeste (Olivera y Yacobaccio 1999); norte de Chubut (Gómez Otero et al. 2000) y Tierra del Fuego (Yesner et al. 1991; Borrero et al. 2001; Guichón et al. 2001).
Con la finalidad de comparar con otras regiones de la Argentina, hemos elaborado un gráfico (Figura 6) en donde podemos observar los valores de $\delta^{13} \mathrm{C}$ y $\delta^{15} \mathrm{~N}$ para distintas áreas elegidas. Los valores más negativos de $\delta^{13} \mathrm{C}$ y los más bajos $\delta^{15} \mathrm{~N}$ señalarían una dieta proteica de origen terrestre con influencias de recursos $C_{3}$, como por ejemplo algunos individuos de Tierra del Fuego (ver cuadrante inferior izquierdo de la figura 6). Los valores menos negativos de $\delta^{13} \mathrm{C}$ y los más bajos $\delta^{15} \mathrm{~N}$ señalan una dieta proteica de origen terrestre con influencias de recursos $\mathrm{C}_{4}$, como sería el caso de los individuos del noroeste argentino (cuadrante inferior derecho). Cuando los valores de $\delta^{13} \mathrm{C}$ son menos negativos y los de $\delta^{15} \mathrm{~N}$ más elevados, estamos en presencia de una dieta marítima, como por ejemplo algunos individuos de Tierra del Fuego (cuadrante superior derecho). Las muestras de la Puna de Jujuy y de la localidad arqueológica de Río Doncellas se ubican alrededor del centro de la gráfica y sus valores son intermedios para el $\delta^{13} \mathrm{C}$ y mayores al $12 \%$ para el $\delta^{15} \mathrm{~N}$.

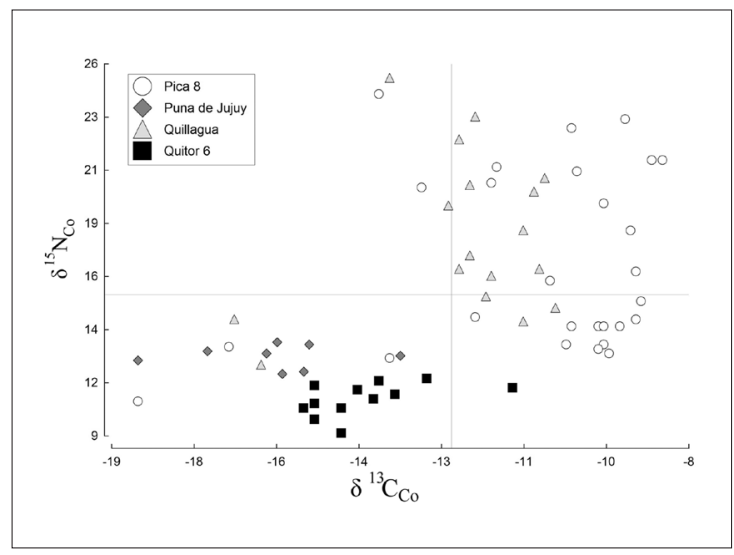

Figura 7. Representación gráfica de los valores de $\delta^{13} C_{y} \delta_{15} \mathrm{Nco}$ de distintos sitios arqueológicos del período Intermedio Tardío del desierto de Atacama como Quillagua (Pestle et al. 2015); Pica 8 y Quitor 6 (Santana-Sagredo et al. 2015) y la Puna de Jujuy (muestra actual).

Además, se compararon los resultados obtenidos en este trabajo con las poblaciones locales del período Intermedio Tardío del desierto de Atacama (Figura 7). Entre ellas, la población de Quillagua manifestó altos valores de nitrógeno que sugieren un alto consumo de proteínas de origen marino. Cabe destacar que este sitio se encuentra a $70 \mathrm{~km}$ de la costa (Pestle et al. 2015). La población de Pica 8 manifestó una dieta consistente con el consumo 
de maíz y de los recursos marinos. Presentaron una media de $-10,9 \%$ para el $\delta^{13} \mathrm{C}$ y $17 \%$ para el $\delta^{15} \mathrm{~N}(\mathrm{n}=27)$ (Santana Sagredo et al. 2015). En Quitor 6, los individuos manifestaron una dieta terrestre que comprendía recursos $\mathrm{C}_{3}$ y $\mathrm{C}_{4}$, con una contribución importante del maíz. Presentaron una media de $-14,4 \%$ para el $\delta^{13} \mathrm{C}$ y $11 \%$ para el $\delta^{15} \mathrm{~N}(\mathrm{n}=13)$. Los valores elevados del nitrógeno se explican por el uso de abono y/o por la aridez de la región (Santana Sagredo et al. 2015). Los valores isotópicos de la Puna de Jujuy son muy parecidos a esta última población, manifestando sus mismas características y problemáticas.

En el valle de Ayacucho, Perú, se realizó un trabajo en donde se dieron a conocer los valores isotópicos del $\delta^{13} \mathrm{C}$ y $\delta^{15} \mathrm{~N}$ del colágeno óseo, músculo y piel de varias momias del período colonial (Finucane 2007). Los resultados arrojaron una media de -11,5\% para el $\delta^{13} \mathrm{C}$ y $11,1 \%$ o para el $\delta^{15} \mathrm{~N}$ para el colágeno óseo, estos datos indican que el maíz cumplía un rol muy importante en la subsistencia de estos individuos. Además, la significativa correlación obtenida entre los valores de $\delta^{13} \mathrm{C}$ y $\delta^{15} \mathrm{~N}$ del colágeno óseo implica que hay una misma causa de enriquecimiento de esos valores, el uso de abono animal. Por lo tanto, el maíz fertilizado con estiércol sería enriquecido en $\delta^{13} \mathrm{C}$ y $\delta^{15} \mathrm{~N}$, así como también los tejidos de los individuos que consumen ese maíz (Finucane 2007).

\section{$*$ Conclusiones}

Consideramos que este trabajo constituye un primer acercamiento a la dieta de los individuos puñenos en el período Tardío en su primera etapa de la vida. Los valores de $\delta^{13} \mathrm{C}$ de la mayoría de los individuos indicaron que se habría mantenido una dieta mixta y los valores del $\delta_{15}$ Nco se manifestaron elevados en los distintos sitios. Este alto porcentaje de los valores del nitrógeno puede ser explicado por el uso de abono en los campos de cultivo o la aridez del clima. Estos resultados fueron coherentes con los datos publicados para la misma región y para zonas aledañas, como San Pedro de Atacama.

En una instancia futura será necesario ampliar la muestra, realizar comparaciones entre los valores isotópicos del $\delta^{13} \mathrm{C}$ y del $\delta^{15} \mathrm{~N}$ para el colágeno y la apatita, para así establecer los hábitos alimentarios tanto de proteínas como de carbohidratos y grasas. También nos interesa explorar si la etapa juvenil es similar a la etapa adulta de los individuos puneños, a través de la información que reflejan los huesos y los dientes de un mismo individuo. Además, se requiere de una minuciosa documentación de la ecología isotópica de la Puna de Jujuy con el fin de avanzar en una interpretación más precisa.

Agradecimientos Queremos agradecer a las siguientes instituciones: Consejo Nacional de Investigaciones Científicas y Técnicas (PIP 2405/08); Secretaría de Ciencia y Técnica, Universidad Nacional de Río Cuarto (SeCyTUNRC. C437/13); Agencia Nacional de Promoción Científica y Técnica y FONCyT (PICT No 02210/07). También nuestro agradecimiento a Myriam Tarragó y a Claudia Aranda, del Museo etnográfico, a Héctor Pucciarelli y a Mariano Del Papa, del Museo de La Plata, a Mitzi di Martino y Greg Hodgins del NSF Arizona AMS Facility y a Julián Valetti.

\section{* Referencias citadas}

ALBECK, M. E. 1998. Pueblo Viejo de Tucute: Sorcuyo revisitado, una nueva visión sobre un sitio clásico del Noreste Argentino. Chungara 30(2): 143-160.

2011. Estudios de agricultura prehispánica en Casabindo (1980-1993). En Arqueología de la Agricultura. Casos de estudio en la región andina argentina, M. A. Korstanje y M. Quesada (Eds.), pp. 12-47. Ediciones Magna, Santiago.

ALBECK, M. E. y M. RUÍZ. 2003. El tardío en la Puna de Jujuy: poblados, etnias y territorios. Cuadernos FHyCS-UNJu 20: 199221.
ALBECK, M. E. y M. A. ZABURLÍN. 2008. Aportes a la cronología de los asentamientos agropastoriles de la Puna de Jujuy. Relaciones de la Sociedad Argentina de Antropología 33: 155-180.

ALFARO, L. C. 1983. Investigación arqueológica en la cuenca del Río Doncellas (Provincia de Jujuy). Integración de la Puna Jujeña a los centros culticos andinos. Relaciones de la Sociedad Argentina de Antropología 15: 25-47.

1988. Investigación Arqueológica en la Cuenca del río Doncellas. Dpto. Cochinoca, Jujuy. Reconstrucción de una cultura olvidada en la Puna Jujeña. Talleres Gráficos del Boletín Oficial e Imprenta del 
Estado de la Provincia de Jujuy, San Salvador de Jujuy.

ALFARO, L. C.y J. M. SUETTA. 1970. Nuevos aportes para el estudio del asentamiento humano en la Puna de Jujuy. Revisión del Pucará de Rinconada. Antiquitas 10: 1-10.

1976. Excavaciones en la cuenca del río Doncellas. Antiquitas 22-23: 1-32.

AMBROSE, S. H. 1990. Preparation and characterization of bone and tooth collagen for isotopic analysis. Journal of Archaeological Science 17: 431-451.

1991. Effects of diet, climate and physiology on nitrogen isotope abundances in terrestrial foodwebs. Journal of Archaeological Science 18: 293-317.

1993. Isotopic analysis of paleodiets: methodological and interpretive considerations. Investigacions of Ancient Human Tisuee 59-130.

AMBROSE, S. H. y B. M. BUTLER. 1997. Stable isotopic analysis of human diet in the Marianas Archipelago, Western Pacific. American Journal of Physical Anthropology 104(3): 343-361.

AMBROSE, S. H., J. BUIKSTRAy H. W. KRUEGER. 2003. Status and gender differences in diet at Mound 72, Cahokia, revealed by isotopic analysis of bone. Journal of Anthropological Archaeology 22: $217-226$.

AMBROSE, S. H. y L. NORR. 1993. Experimental evidence for the relationship of the carbon isotope ratios of whole diet and dietary protein to those of bone collagen and carbonate. En Prehistoric human bone: Archaeology at the Molecular Level, J. B. Lambert y G. Grupe (Eds.), pp. 1-37. Springer-Verlag, Berlin.

BOMAN, E. 1908. Antiquitiés de la région andine de la République Argentine et du désert d'Atacama, 1. Imprimerie nationale, H. Le Soudier.

BORRERO, L. A., R. GUICHÓN, R. TIKOT, J. KELlY, A. PRIETO y P. CÁRDENAS. 2001. Dieta a partir de isótopos estables en restos óseos humanos de Patagonia Austral. Estado actual y perspectivas. Anales Instituto de la Patagonia 29: 119-127.

BUIKSTRA, J. E. y G. R. MILNER. 1991. Isotopic and archaeological interpretations of diet in the Central Mississippi Valley. Journal of Archaeological Science 18(3): 319-329.

CASANOVA, E. 1938. Investigaciones arqueológicas en Sorcuyo, Puna de Jujuy. Anales del Museo Argentino de Ciencias Naturales $80(39): 423-462$.
COLTRAIN, J. B. y S. W. LEAVITT. 2002. Climate and diet in Fremont prehistory: economic variability and abandonment of maize agriculture in the Great Salt Lake Basin.American Antiquity 67: 453-485.

DeNIRO, M. J. 1987. Stable isotopy and archaeology. American Scientist 182-191.

FERNÁNDEZ, J.y H. O. PANARELLO. 1999-2001a. Los isótopos estables del carbono en pelo de animales silvestres de ambientes altiplánicos de Argentina. Xama 12-14: 61-69.

1999-2001b. Isótopos del carbono en la dieta de herbívoros y carnívoros de los andes jujeños. Xama 12-14: 71-85.

FINUCANE, B. C. 2007. Mummies, maize, and manure: multitissue stable isotope analysis of late prehistoric human remains from the Ayacucho Valley, Peru. Journal of Archaeological Science 34(12): 2115-2124.

FOGEL, M., N. TUROS y D. W. OWSLEY. 1989. Nitrogen isotope tracers of human lactation in modern and archaeological populations. Annual report of the Director; Geophysical Laboratory. Washington, D. C.: Carnegie Institution, pp.: 111-117.

FUCHS, M. L. y H. H. VARELA. 2013. Fechados radiocarbónicos de colecciones osteológicas de Puna de Jujuy, Argentina. Relaciones de la Sociedad Argentina de Antropología XXXVIII (2): 553-558.

GÓMEZ OTERO, J., J. B. BELARDI, R. TIKOT y S. GRAMMER. 2000. Dieta y poblaciones humanasen la costa norte del Chubut (Patagonia Argentina). En Desde el País de Los Gigantes. Perspectivas arqueológicas en Patagonia, pp. 109-122. Universidad Nacional de la Patagonia, Río Gallegos, Santa Cruz.

GUICHÓN, R., L. BORRERO, A. PRIETO, P. CÁRDENAS y R. TIKOT. 2001. Nuevas determinaciones de isótopos estables para Tierra del Fuego. Revista Argentina de Antropología Biológica 3(1): 113-126.

HEATON, T. H. E., J. C. VOGEL, G. VON LA CHEVALLIERIE y G. COLLETT. 1986. Climatic influence on the isotopic composition of bone nitrogen. Nature 322: 822-823.

KATZENBERG, M. A. 1999. A re-examination of factors contributing to elevated stable nitrogen isotope values in infants and young children. American Journal of Physical Anthropology 28 (suppl): 165 .

2008. Stable isotope analysis: tool for studying past diet, demography, and life history. En Biological Anthropology of Human Skeleton, M. A. Katzemberg y S. R. Saunders (Eds.), pp. 413-441. John Wiley \& Sons, Hoboken. 
KATZENBERG, M. A. y A. WEBER. 1999. Stable isotope ecology and paleodieta in the Lake Baikal region of Siberia. Journal of Archaeological Science 26: 651-665.

KATZENBERG, M. A., H. P. SCHWARCZ, M. KNYFy F. J. MELBYE. 1995. Stable isotope evidence for maize horticulture and paleodiet in southern Ontario, Canada. American Antiquity 60: 335-350.

KATZENBERG, M. A. y N. C. LOVELL. 1999. Stable isotope variation in pathological bone. International Journal Osteoarchaeology 9:316-324.

KILLIAN GALVÁN, V. A., D. E. OLIVERAy E. GALLEGOS. 2012. Una aproximación isotópica al consumo de maíz en la localidad arqueológica Río Doncellas (Depto. De Cochinoca, Prov. de Jujuy). En Las manos en la masa. Arqueologías, Antropologías e Historias de la Alimentación en Suramérica, M. P. Babot, M. Marschoff y F. Pazzarelli (Eds.), pp. 319-338. Museo de Antropología, Universidad Nacional de Córdoba, Córdoba.

KILLIAN GALVÁN, V. A. y P. SALMINCI. 2014. Aportes a la ecología isotópica: información actual y sistemas de regadío arqueológicos en la microrregión de Antofagasta de la Sierra (provincia de Catamarca, Argentina). Comechingonia 18(1): 51-72.

KNUDSON, K. J., T. D. PRICE, J. E. BUIKSTRA y D. E. BLOM. 2004. The use of strontium isotope analysis to investigate Tiwanaku migration and mortuary ritual in Bolivia and Peru. Archaeometry 46: 5-18.

LEHMANN NITSCHE, R. 1902. Catálogo de las antigüedades de la Provincia de Jujuy. Revista del Museo de La Plata XI: 75-12O.

MADRAZO, G. y M. OTTONELLO DE GARCÍA REYNOSO. 1966. Tipos de instalación prehispánica en la región de la Puna y su borde. En Monografías I, Museo Etnográfico Municipal “Dámaso Arce", Olavarría, Buenos Aires.

MINAGAWA, M. y E. WADA. 1984. Stepwise enrichment of ${ }_{15} \mathrm{~N}$ along food chains: further evidence and the relation between $\delta_{15} \mathrm{~N}$ and animal age. Geochim Cosmochim Acta 48: 1135-1140.

NELSON, S. J. y M. M. ASH. 2010. Wheeler's dental anatomy, physio$\log y$ and occlusion. Saunders, St. Louis.

NIELSEN, A. E. 1997-1998. Tráfico de caravanas en el sur de Bolivia: observaciones etnográficas e implicancias arqueológicas. Relaciones de la Sociedad Argentina de Antropología 22-23: 139-178.

OLIVERA, D. E. y H. D. YACOBACCIO. 1999. Estudios de paleodieta en poblaciones humanas de los Andes del sur a través de isótopos estables. Trabajo presentado al V Congreso Nacional de Paleopatología, Alcalá La Real, Jaén.
OTTONELLO DE GARCÍA REINOSO, M. 1973. Instalación, economía y cambio cultural en el sitio tardío de Agua Caliente de Rachaite. Publicaciones 1: 23-68.

PANARELLO, H. O., A. TESSONE y A. F. J. ZANGRANDO. 2006-2009. Isótopos estables en arqueología: principios teóricos, aspectos metodológicos y aplicaciones en Argentina. Xama 19-23: 115-133.

PATE, F. D. 1994. Bone chemistry and paleodiet. Journal of Archaeological Method and Theory 1: 161-209.

PATE, F. D., J. L. CRAIB y G. M. HEATHCOTE. 2ool. Stable isotopic analysis of prehistoric human diet in the Mariana Islands, western Pacific. Australian Archaeology 52: 1-4.

PÉREZ, M. y V. A. KILLIAN GALVÁN. 2011. Doncellas (Puna Septentrional, Jujuy, Argentina): nuevos enfoques a partir del estudio cerámico y del análisis paleodietario. Estudios Atacameños 42: 79-100.

PESTLE, W. J., C. TORRES-ROUFF, M. HUBBE, F. SANTANA, G. PIMENTEL, F. GALLARDO y K. L. KNUDSON. 2015. Explorando la diversidad dietética en la prehistoria del desierto de Atacama: un acercamiento a los patrones regionales. Chungara, Revista de Antropología Chilena 47(2): 201-209.

POULSON, S. R., S. C. KUZMINSKY, G. R. SCOTT, V. G. STANDEN, B. ARRIAZA, I. MUÑOZ y L. DORIO. 2013. Paleodiet in northern Chile through the Holocene: extremely heavy $\delta_{15} \mathrm{~N}$ values in dental calculus suggest a guano-derived signature? Journal of Archaeological Science 40(12): 4576-4585.

SANTANA-SAGREDO, F., J. A. LEE-THORP, R. SCHULTING y M. URIBE. 2015. Isotopic evidence for divergent diets and mobility patterns in the Atacama Desert, northern Chile, during the Late Intermediate Period (AD 900-1450). American Journal of Physical Anthropology 156(3): 374-387.

SCHOENINGER, M. J. y M. J. DeNIRO. 1984. Nitrogen and carbon isotopic composition of bone collagen from marine and terrestrial animals. Geochim Cosmochim Acta 48: 625-639.

SCHWARCZ, H. P. y M. J. SCHOENINGER. 1991. Stable isotope analyses in human nutritional ecology. Yearbook of Physical Anthropoogyl 34: 283-322.

SCHWARCZ, H. P., T. L. DUPRAS y S. I. FAIRGRIEVE. 1999. 15N enrichment in the Sahara: in search of a global relationship. Journal of Archaeological Science 26(6): 629-636.

SEALY, J. C., N. J. VAN DER MERWE, A. K. F. J. SILLEN y H. W. KRUEGER. 1991. 87Sr/86Sr as a dietary indicator in modern and archaeological bone. Journal of Archaeological Science 18:399-416. 
SEALY, J. C., N. J. VAN DER MERWE, J. A. THORP y J. L. LANHAM. 1987. Nitrogen isotopic ecology in southern Africa: implications for enviromental and dietary tracing. Geochim Cosmochim Acta 51: 2707-2717.

SZPAK, P., J. F. MILLAIRE, C. D. WHITE y F. J. LONGSTAFFE. 2012. Influence of seabird guano and camelid dung fertilization on the nitrogen isotopic composition of fieldgrown maize (Zea mays). Journal of Archaeological Science 39(12): 3721-3740.

TYKOT, R.H. 2006. Isotope Analyses and the Histories of Maize. En Histories of maize, J.E. Staller, R.H. Tykot y B.F. Benz (Eds.), pp. 131-142. Elsevier, Amsterdam.

VAN DER MERWE, N., R. F. WILLIAMSON, S. PFEIFFER, S. C. THOMAS y K. O. ALLEGRETTO. 2003. The Moatfield ossuary: isotopic dietary analysis of an Iroquoian community, using dental tissue. Journal of Anthropological Archaeology 22: 245-261.

VOGEL, J. C. y N. VAN DER MERWE. 1977. Isotopic evidence for early maize cultivation in New York State. American Antiquity 42: $238-242$.

WHITE, C. D. y G. J. ARMELAGOS. 1997. Osteopenia and stable isotope ratios in bone collagen of Nubian female mummies. American Journal of Physical Anthropology 103: 185-199.
WHITE, C. D., M. W. SPENCE, H. L. Q. STUART-WILLIAMS y H. P. SCHWARCZ. 1998. Oxygen isotopes and the identification of geographical origins: the Valley of Oaxaca versus the Valley of Mexico. Journal of Archaeological Science 25: 643-655.

YACOBACCIO, H. D., M. R. MORALES y C. T. SAMEC. 2009. Towards an isotopic ecology of herbivory in the Puna ecosystem: news results and patterns on lama glama. International Journal of Osteoarchaeology 19: 144-155.

YACOBACCIO, H. D., C. T. SAMEC y M. P. CATÁ. 2010. Isótopos estables y zooarqueología de camélidos en contextos pastoriles de la puna (Jujuy, Argentina). En Zooarqueología a principios del siglo XXI: Aportes teóricos, metodológicos y casos de estudio, M. Gutiérrez, M. De Nigris, P. Fernández, M. Giardina, A. Gil, A. Izeta, G. Neme y H. Yacobaccio (Eds.), pp. 77-86. Ediciones del Espinillo, Buenos Aires.

YESNER, D., M. FIGUERERO TORRES, R. GUICHÓN y L. A. BORRERO. 1991. Análisis de isótopos estables en esqueletos humanos: confirmación de patrones de subsistencia etnográficos para Tierra del Fuego. Shincal 3(2): 182-191.

YESNER, D. R., M. J. F. TORRES, R. A. GUICHÓN y L. A. BORRERO. 2003. Stable isotope analysis of human bone and ethnohistoric subsistence patterns in Tierra del Fuego. Journal of Anthropological Archaeology 22: 279-291. 